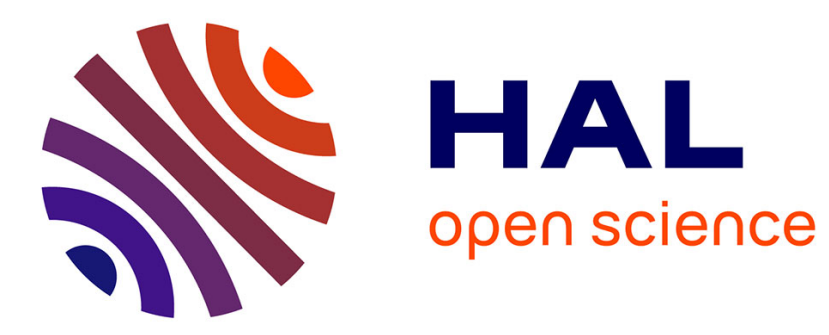

\title{
Magnetostriction of spin glass alloys
}

G. Creuzet, I.A. Campbell

\section{- To cite this version:}

G. Creuzet, I.A. Campbell. Magnetostriction of spin glass alloys. Journal de Physique Lettres, 1982, 43 (15), pp.575-582. 10.1051/jphyslet:019820043015057500 . jpa-00232094

\section{HAL Id: jpa-00232094 https://hal.science/jpa-00232094}

Submitted on 1 Jan 1982

HAL is a multi-disciplinary open access archive for the deposit and dissemination of scientific research documents, whether they are published or not. The documents may come from teaching and research institutions in France or abroad, or from public or private research centers.
L'archive ouverte pluridisciplinaire HAL, est destinée au dépôt et à la diffusion de documents scientifiques de niveau recherche, publiés ou non, émanant des établissements d'enseignement et de recherche français ou étrangers, des laboratoires publics ou privés. 
Classification

Physics Abstracts

75.80

\title{
Magnetostriction of spin glass alloys
}

\author{
G. Creuzet and I. A. Campbell \\ Laboratoire de Physique des Solides, Bâtiment 510, \\ Université Paris-Sud, Centre d'Orsay, 91405 Orsay, France
}

(Reçu le 18 mai 1982, révisé le 11 juin, accepté le 16 juin 1982)

\begin{abstract}
Résumé. - Nous présentons des mesures de magnétostriction de volume et de forme sur des alliages $\mathrm{CuMn}, \mathrm{AuFe}$ et AgMn à diverses concentrations. Le paramètre de Gruneisen effectif déduit de l'effet

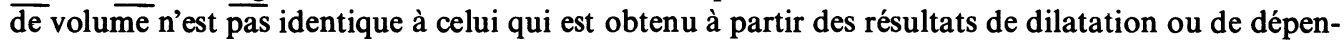
dance en pression de la température $T_{\mathrm{g}}$. L'influence des différentes interactions, RKKY et à courte portée, constitue la base de notre discussion. Aucune contribution de forme n'est présente dans les séries $\mathrm{CuMn}$ et $\mathrm{AgMn}$, à l'opposé des alliages $\mathrm{AuFe}$ où un tel effet est clairement observé. Ce résultat indique la présence de moment orbital sur les atomes de $\mathrm{Fe}$ dans ces systèmes. Enfin, nous pouvons obtenir des informations sur les processus d'aimantation dans ces alliages à partir de la dépendance en champ de nos mesures.
\end{abstract}

\begin{abstract}
We have measured the volume and shape magnetostriction of $\mathrm{CuMn}, \mathrm{AuFe}$ and $\mathrm{AgMn}$ alloys. The effective Gruneisen parameter deduced from the volume magnetostriction is not the same as that obtained from thermal expansion or from the pressure dependence of the spin glass temperature $T_{\mathbf{g}}$. We discuss this in terms of the influence of RKKY and shorter range interactions. We have found no shape magnetostriction in CuMn and AgMn while a definite effect can be seen in AuFe. This indicates a local orbital moment on Fe in this alloy. From the field dependence of this effect we can obtain information on the magnetization processes in these alloys.
\end{abstract}

1. Introduction - From magnetostriction measurements one can obtain information on the volume dependence and strain dependence of magnetic energy terms. We have applied this technique to a number of spin glass alloy systems; from the results we can draw conclusions about the magnetic character of these alloys and about the magnetization processes.

We have measured the low temperature magnetostriction of $\underline{\mathrm{CuMn}}$, $\underline{\mathrm{AuFe}}$ and AgMn polycrystalline samples using a capacity bridge technique. Most of the measurements were performed at $4.2 \mathrm{~K}$ which is well below the spin glass temperature $T_{\mathrm{g}}$ in every case. Magnetic fields up to $70 \mathrm{kG}$ could be applied along the measuring direction or up to $40 \mathrm{kG}$ perpendicular to the measuring direction. The results can be expressed in terms of volume and shape magnetostrictions, defined as :

$$
\frac{\Delta V}{V}(H)=\left(\frac{\delta l}{l}\right)_{\|}(H)+2\left(\frac{\delta l}{l}\right)_{\perp}(H)
$$


and

$$
\left(\frac{\delta l}{l}\right)^{*}(H)=\left(\frac{\delta l}{l}\right)_{\|}(H)-\frac{1}{3} \frac{\Delta V}{V}(H) .
$$

Results are shown in figures 1 through 5. Volume terms were positive for all samples. For the $\mathrm{CuMn}$ and AgMn series the shape magnetostriction was zero within the sensitivity of our system (i.e. $\left.\mid(\delta l / l)^{*} T \lesssim 10^{-8}\right)$ while the AuFe samples showed distinct shape effects - the shape term actually dominating the volume term at the higher concentrations.

2. Volume magnetostriction - We will begin by discussing the volume term. The volume magnetostriction is related to the pressure dependence of the magnetization through the Maxwell relation :

$$
\frac{1}{V} \frac{\partial V}{\partial H}=-\frac{\partial M}{\partial P}
$$

Now in these spin glass systems, other information already exists about certain types of pressure effects, or equivalently of volume dependences. Thus, conventionally, the ratio of the magnetic thermal expansion to the magnetic specific heat is given in terms of a "magnetic Gruneisen factor $» \gamma_{m}$ expressing the change of the magnetic energy with volume; $\gamma_{m}$ is defined by [1] :

$$
\gamma_{\mathrm{m}}(T)=3 B V_{0} \alpha_{\mathrm{m}}(T) / C(T)
$$

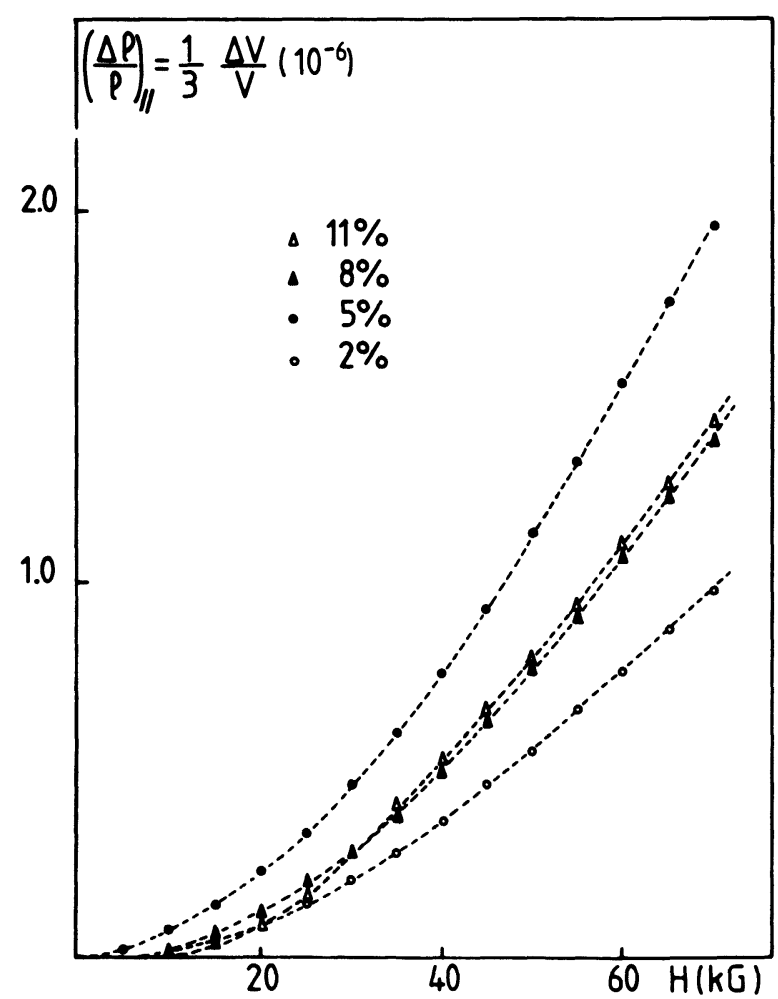

Fig. 1. - Isotropic magnetostriction of polycrystalline $\mathrm{CuMn}$ dilute alloys for several concentrations at $T=4.2 \mathrm{~K}$. Concentrations are in atomic $\%$ throughout. 


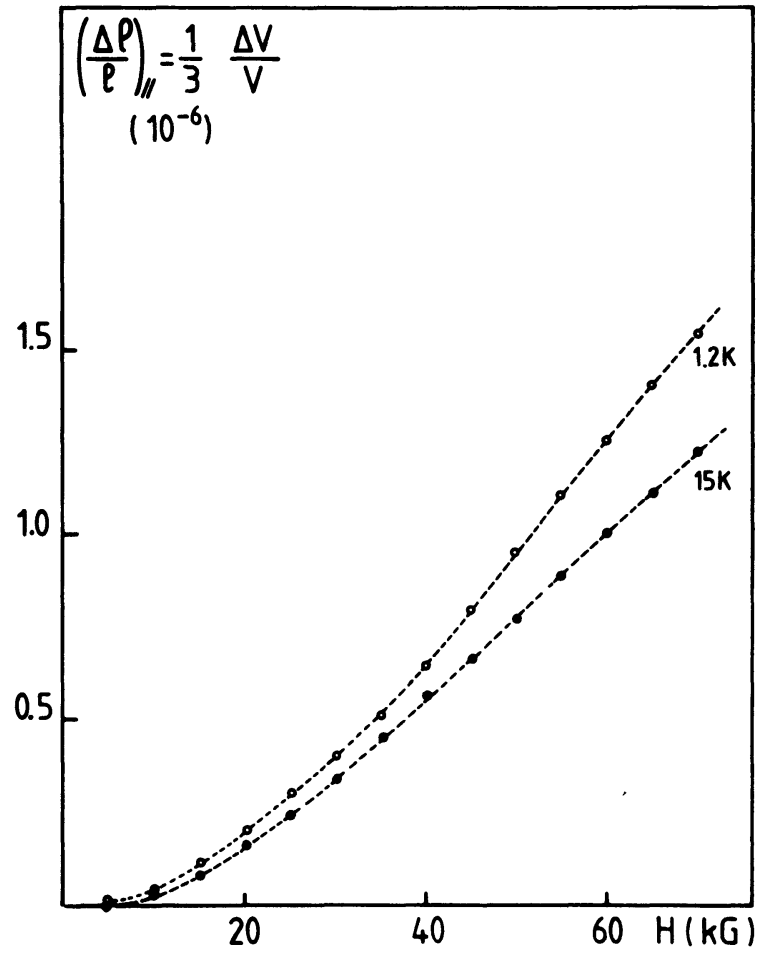

Fig. 2. - Isotropic magnetostriction of polycrystalline $\mathrm{AgMn}, c=2.5 \%$, at representative temperatures in the spin glass $(T=1.2 \mathrm{~K})$ and the paramagnetic $(T=15 \mathrm{~K})$ regions.

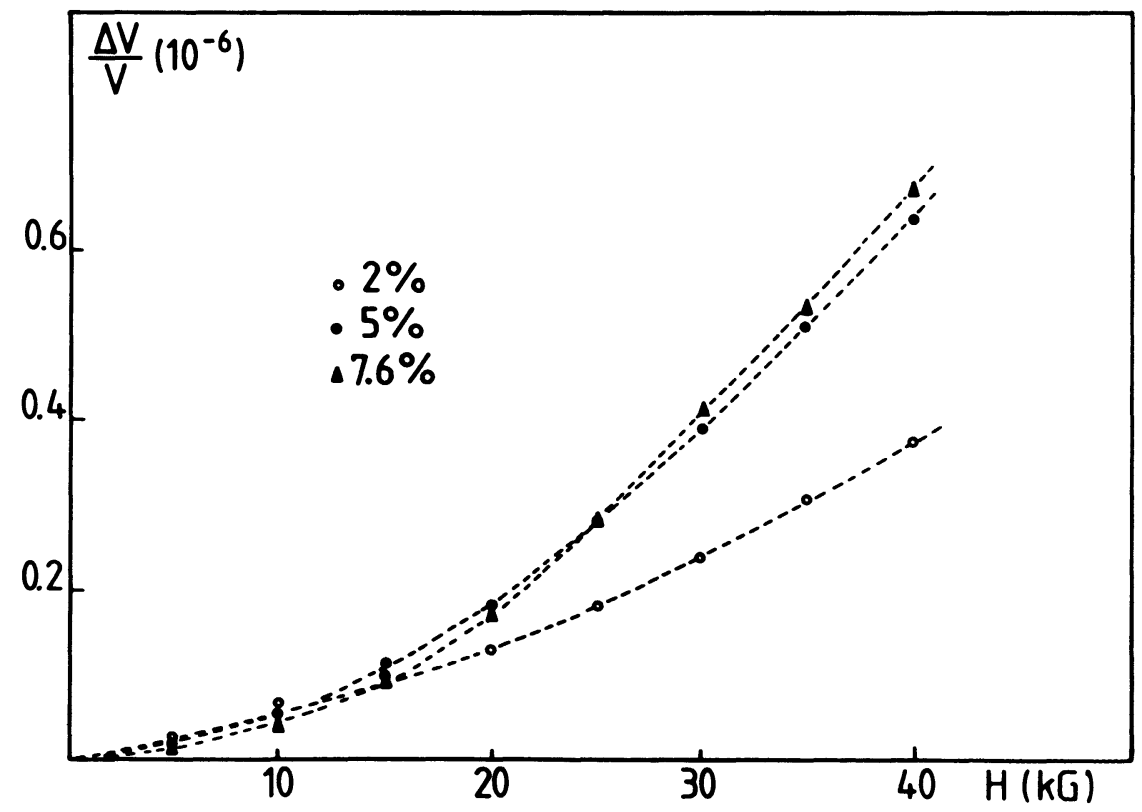

Fig. 3. - Volume magnetostriction of polycrystalline $\underline{\mathrm{AuFe}}$ dilute alloys for several concentrations at $T=4.2 \mathrm{~K}$. 


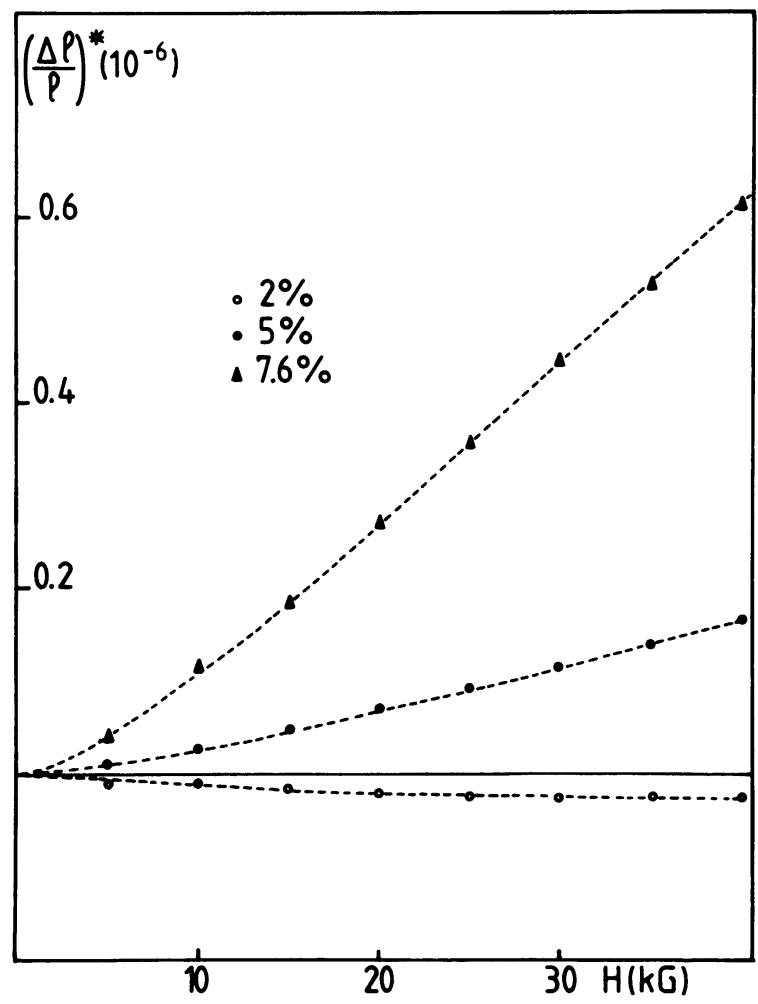

Fig. 4. - Shape magnetostriction of polycrystalline $\underline{\mathrm{AuFe}}$ dilute alloys for several concentrations at $T=4.2 \mathrm{~K}$.

where $B$ is the elastic constant $\left(\left(C_{11}+2 C_{12}\right) / 3\right)$,

$V_{0}$ is the molar volume,

$\alpha_{\mathrm{m}}(T)$ is the magnetic linear thermal expansion, and,

$C(T)$ is the magnetic specific heat.

For a number of CuMn and AgMn alloys $\gamma_{\mathrm{m}}$ has been measured by Kahn and Griffiths [2]

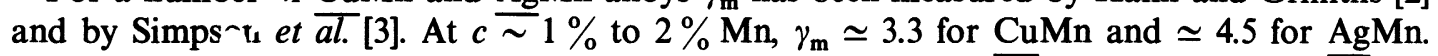
Simpson et al. [3] suggest that $\gamma_{\mathrm{m}}$ is actually temperature dependent; the values we quote are for $T \sim T_{\mathrm{g}}$ and $\gamma_{\mathrm{m}}$ appears to drop somewhat at lower $T$.

Now we can equally well define a magnetostriction Gruneisen parameter :

$$
\gamma_{\mathrm{m}}^{*}=\frac{\mathrm{d} \ln M}{\mathrm{~d} P} / \frac{\mathrm{d} \ln V}{\mathrm{~d} P}=B V_{0}\left[\frac{1}{V} \frac{\partial V}{\partial H} / M(H)\right]
$$

where $M(H)$ is the magnetization per mole. Thermodynamically, $\gamma_{m}^{*}$ and $\gamma_{m}$ are quite independent quantities [4].

This compares the magnetostrictive volume change to the change in internal magnetic energy produced by the applied field, and expresses the volume dependence of this magnetic energy term. We have estimated $\gamma_{\mathrm{m}}^{*}$ for the alloys we have worked on by using our magnetostriction data together with magnetization results on the same alloys from the literature (Smit et al. [5], Bouchiat [6] and Tholence [7]). For more accurate estimates it would be preferable to make the two types of measurement on the same samples, and to obtain values at different temperatures. 


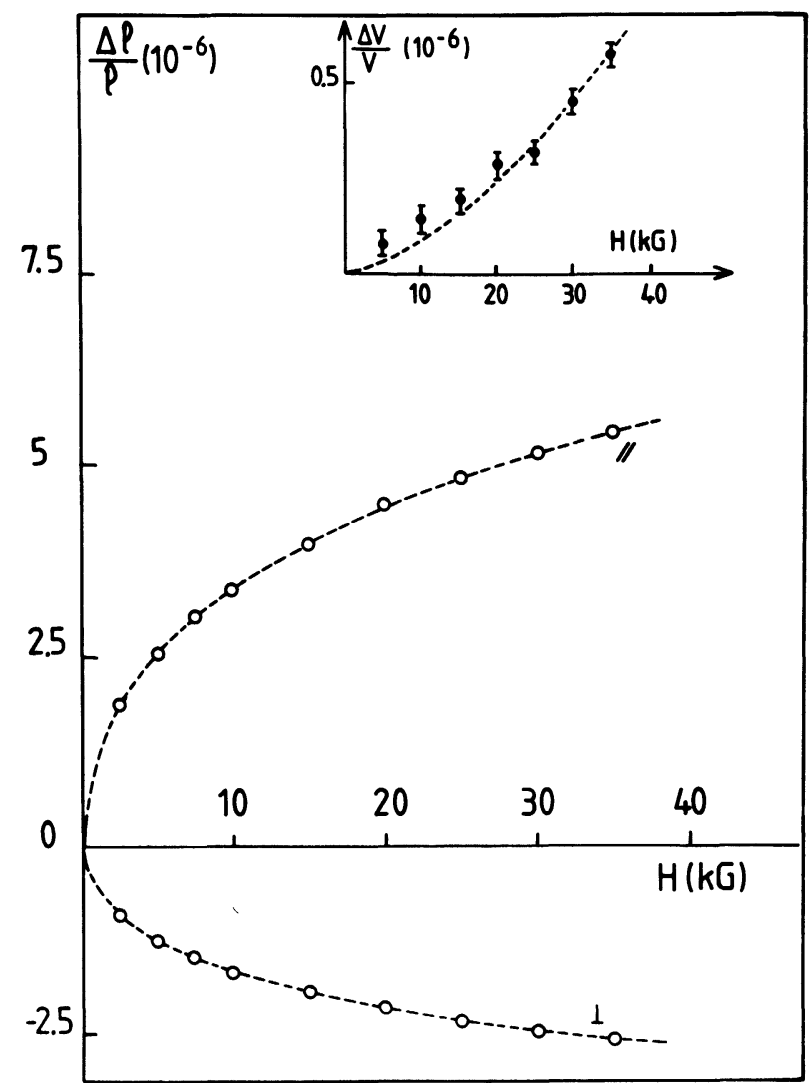

a)

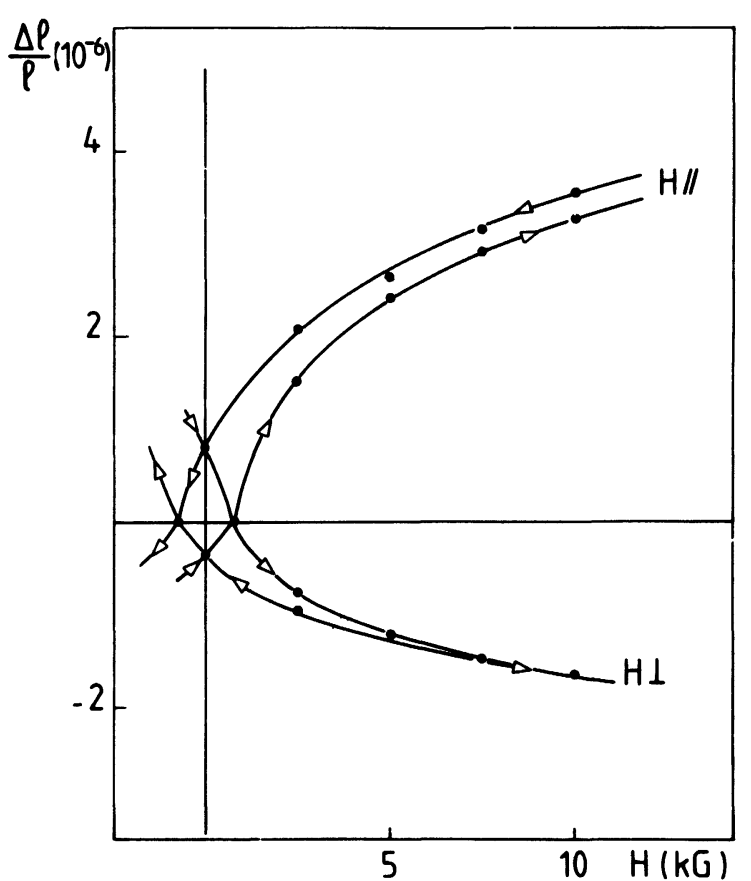

b)

Fig. 5. - (a) Longitudinal and transverse magnetostriction of polycrystalline $\mathrm{AuFe}, c=14 \%, T=4.2 \mathrm{~K}$. Curves shifted along the $H$ axis to correct for hysteresis. In insert : volume part of the effect. (b) Details of the hysteresis in the recording of the magnetostriction near $H=0$. Note that in figure $5 a$ the curves are shifted to $H=0$ for $\Delta l / l=0$.

For a number of spin glass alloys, Schilling [8] has compared the following parameters :

i) $\gamma_{\mathrm{m}}$

ii) $-\mathrm{d} \ln \left(T_{\mathrm{g}}\right) / \mathrm{d} \ln (V)$ measured directly

iii) $-\mathrm{d} \ln \left(J_{2}^{2} n\left(E_{\mathrm{F}}\right)\right) / \mathrm{d} \ln V$ estimated from measurements in the Kondo regime. $J_{2}$ is the Kondo term in the local s-d coupling parameter and $n\left(E_{\mathrm{F}}\right)$ is the conduction electron density of states at the Fermi level. We can also include in the comparison the values we have obtained for $\gamma_{\mathrm{m}}^{*}$. Values are given in figure 6 .

If the interactions were determined solely by the RKKY mechanism dominated by a single coupling parameter $J_{2}$, following Schilling we would expect all these parameters to be the same for a given alloy. While agreement is reasonable for AgMn $2 \%$, this is clearly not the case for $\mathrm{CuMn}$ and $\mathrm{AuFe}$ at similar concentrations. We find that for each of the three systems our value of $\gamma_{\mathrm{m}}^{*}$ at $c \sim 2 \%$ is close to Schilling's estimate of $-\mathrm{d} \ln \left(J_{2}^{2} n\left(E_{\mathrm{F}}\right)\right) / \mathrm{d} \ln V$. As Schilling's parameter should be directly related to the Kondo term in long range RKKY interactions, this result suggests that the magnetization process (at moderate applied fields) is controlled by these same long range interactions. For $\mathrm{CuMn}$ and $\mathrm{AuFe}$, the values of $\gamma_{\mathrm{m}}$ and of $-\mathrm{d} \ln \left(T_{\mathrm{g}}\right) / \mathrm{d} \ln V$ are clearly lower than $\gamma_{\mathrm{m}}^{*}$, implying that the ordering temperature $T_{\mathrm{g}}$ and the specific heat around $T_{\mathrm{g}}$ vary with volume in a different way to what would be expected on this simple approach. 


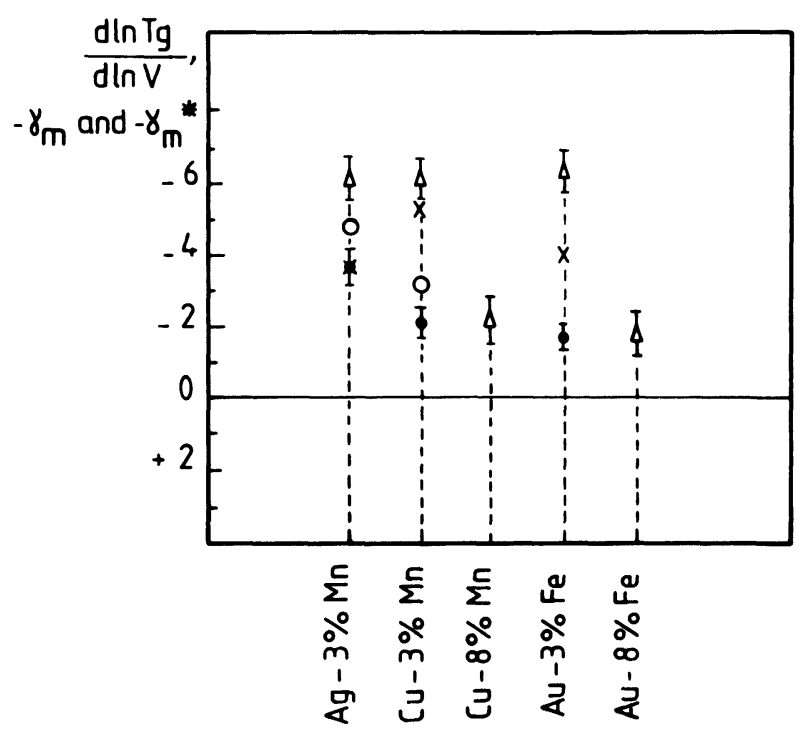

Fig. 6. - Volume dependence of magnetic ordering temperature for various systems (following Schilling, Ref. [8]). 9 Volume dependence of spin glass freezing temperature $T_{\mathrm{g}}$ (Schilling, Ref. [8]). $O$ Results of thermal expansion (Simpson and Smith, Ref. [3]). $\times$ Values of the dependence $\mathrm{d} \ln \left(N\left(E_{\mathrm{F}}\right) J_{2}^{2}\right) / \mathrm{d} \ln (V)$ (see Ref. [8]). $\triangle$ Values added from our work, $\gamma_{\mathrm{m}}^{*}$. The concentrations quoted are approximate, we have grouped results from different experiments at similar concentrations.

This indicates that these parameters are partly controlled by other interactions : perhaps by other $J_{l}$ terms [6], or possibly by shorter range interactions.

At higher concentrations $\left(c \sim 7\right.$ or $8 \%$ ) we find a value of $\gamma_{\mathrm{m}}^{*}$ in $\mathrm{CuMn}$ and AuFe which is considerably lower than at $c \sim 2 \%$; this higher concentration $\gamma_{\mathrm{m}}^{*}$ is close to the values of $\gamma_{\mathrm{m}}$ and of $-\mathrm{d} \ln \left(T_{\mathrm{g}}\right) / \mathrm{d} \ln (V)$ already quoted. Although it would be more convincing if we had values for these parameters at the higher concentrations, this observation is consistent with the conjecture we have given above: at high concentrations, long range interactions will no longer play an important role, so we would expect all parameters to group around a value appropriate to the short range interactions.

We can note that $-\mathrm{d} \ln T_{\mathrm{g}} / \mathrm{d} \ln V$ is higher for $\underline{\mathrm{AuFe}} 20 \%$ than for AuFe $3 \%$ [6], but the type of ordering in the two systems is not the same.

3. Shape magnetostriction - Shape magnetostriction in dilute magnetic alloys can be expected to be dominated by single ion terms which arise because local magnetic moments can be nonspherical because of the spin-orbit interaction. Other terms such as dipole-dipole interactions can be expected to be negligibly small.

The shape term should be essentially zero in systems where the local magnetic moment has no orbital component as there will then be no anisotropic coupling terms between the moments and the lattice. We have indeed found no observable shape magnetostriction in the two Mn alloy systems CuMn and AgMn which is consistent with zero orbital moment on the Mn sites.

In $\mathrm{Au} \overline{\mathrm{Fe}}$, on the other hand, there is a definite shape effect, (Figs. 4 and 5) which is weak and negative at low concentrations, becoming positive and rapidly stronger at higher concentrations. This is clear evidence for the presence of an orbital moment component on Fe in Au. However the detailed interpretation of the shape magnetostriction as a function of field and concentration in these systems is not straightforward. 
The only well established cases of single ion shape magnetostriction in dilute transition metal alloys are those of PdNi and PdCo (Hölscher and Franse [9] and Creuzet et al. [10], Tokunaga et al. [11]). For these alloys, which are ferromagnets, the shape magnetostriction at saturation is linear in impurity concentration up to $c \simeq 20 \%$. We will assume that this would be the case for $\mathrm{AuFe}$ too, if we could saturate the magnetization. Unfortunately for available applied fields we are still rather far from saturation (Smit et al. [5]). Even for AuFe $14 \%$ which is on the brink of

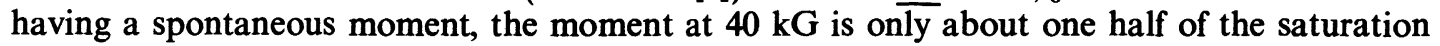
value (J. C. Ousset and H. Rakoto, to be published). Extrapolating the magnetostriction data, we can estimate for the AuFe $14 \%$ sample a saturation magnetostriction $(\delta l / l)_{\text {sat }}^{*} \simeq+15 \mathrm{ppm}$, i.e. $(\delta l / l)_{\text {sat }}^{*} \simeq+1 \mathrm{ppm} / \%$ for AuFe. This can be compared to $(\delta l / l)_{\text {sat }}^{*} \simeq-15 \mathrm{ppm} / \%$ for PdCo, where from hyperfine data [12] we can estimate $\mu_{\text {orb }} \simeq 0.6 \mu_{\mathrm{B}} /$ atom.

No simple relation between the local orbital moment and the magnetostriction for transition metal impurities has been established so far, but we can deduce that the local orbital moment on $\mathrm{Fe}$ in $\mathrm{Au}$ is of the order of a tenth of a $\mu_{\mathrm{B}}$.

The field dependence of the shape magnetostriction gives us new information about the magnetization process. If $\theta$ is the angle between the applied field direction $z$ and the axis of magnetization of a given local moment, the magnetization $M(H, T)$ is proportional to $\mu c\langle\cos \theta\rangle$ while with the assumption of a pure single ion effect the magnetostriction should be proportional to $\lambda c\left\langle 3 \cos ^{2} \theta-1\right\rangle$ where $\lambda$ is the single ion constant. The distribution of $\theta$ in zero field is random; then with increasing applied field $\theta$ values will tend to decrease towards zero. At saturation the magnetostriction will be $2 \lambda c$, but at low field it can have either sign depending on the distribution $P(\theta)$ in the applied field.

For the samples well in the spin glass regime $(2 \%$ and $5 \% \mathrm{Fe})$ the results show that $\left\langle 3 \cos ^{2} \theta-1\right\rangle$ remains small (negative in one case and positive in the other) in fields up to $40 \mathrm{kG}$. This implies a gradual reorientation in field so that the entire distribution $P(\theta)$ shifts towards smaller $\theta$ values. For samples near the critical concentration for ferromagnetism, even in low fields $\left\langle 3 \cos ^{2} \theta-1\right\rangle$ increases rapidly suggesting that the magnetization tends to occur through large angle reorientations of spins or groups of spins to a final spin direction near the $z$ axis ; this is akin to magnetization through domain rotation in a true ferromagnet.

Mössbauer polarization measurements give information which is in principle equivalent to these shape magnetostriction measurements, as there are spectral lines with intensity proportional to $\left\langle 3 \cos ^{2} \theta-1\right\rangle$; in practice for the Mössbauer results the presence of the external field complicates the quantitative analysis. Measurements on AuFe alloys in $50 \mathrm{kG}$ applied fields (Borg [13]) show $\left\langle 3 \cos ^{2} \theta-1\right\rangle$ values which are near zero for Fe concentrations from $2 \%$ to $6 \%$, and which increase rapidly at higher concentrations, in agreement with the present data. We can remark that for the AuFe $2 \%$ the negative effect observed is reminiscent of antiferromagnetic behaviour.

Finally, we should note that the present measurements have been performed on polycrystals. The magnetostriction of dilute alloys can be highly anisotropic (Creuzet [14]) and experiments on single crystals would be necessary to clarify this point for the AuFe case.

4. Conclusion. - The shape magnetostriction results suggest that at $c \sim 2 \%$ the initial magnetization process is controlled principally by the long range RKKY interactions with an effective Gruneisen parameter $\gamma_{\mathrm{m}}^{*}$ of about 6.2 for $\mathrm{CuMn}$, AuFe and AgMn. At higher concentrations for CuMn and AuFe the effective $\gamma_{m}^{*}$ drops, which we ascribe to shorter range interactions becoming dominant. The shorter range interactions play an important role in determining the $T_{\mathrm{g}}$ value even at the $2 \%$ concentration level.

From the shape magnetostriction we can deduce that $\mathrm{Fe}$ in $\mathrm{Au}$ has a local orbital moment of the order of a tenth of a $\mu_{\mathrm{B}}$, while the Mn local moment in CuMn or AgMn is pure spin to within the accuracy of our measurement. The form of the shape magnetostriction curves in the AuFe 
alloy series indicates that well into the spin glass regime the magnetization process is through small angle spin reorientations, while large angle spin reorientations appear to set in when the critical concentration for ferromagnetism is approached.

\section{References}

[1] Barron, T. H. K., Collins, J. G. and White, G. K., Adv. Phys. 29 (1980) 609.

[2] Khan, J. A. and Griffiths, D., J. Phys. F8 (5) (1978) 763.

[3] Simpson, M. A., Smith, T. F., Grelin, E., J. Phys. F 11 (8) (1981) 1655.

[4] Chandrasekar, B. S. and Fawcett, E., Adv. Phys. 20 (1971) 775.

[5] Smit, J. J., Nieuwenhuys, G. J. and DE Jongh, L. J., Solid State Commun. 32 (1979) 233.

[6] Bouchiat, H., Thèse de 3e cycle, Orsay (1981), unpublished.

[7] Tholence, J. L., Thèse d'Etat, Grenoble (1973), unpublished : see also PréJean, J. J., Thèse d'Etat, Grenoble (1980), unpublished.

[8] SCHILling, J. S., The proceedings of the International Symposium on the Physics of Solids under high Pressure, J. S. Schilling and R. N. Shelton, editors (North Holland, Amsterdam) 1981.

[9] Hölscher, H. and Franse, J. J. M., J. Mag. Mag. Mat. 15-18 (1980) 605.

[10] Creuzet, G., Hamzic, A. and Campbell, I. A., Solid State Commun: 39 (1981) 451.

[11] Tokunaga, T., Kohri, M., Kadomatsu, M. and Fujiwara, H., J. Phys. Soc. Japan 50 (1980) 1411.

[12] Katayama, M., Kumagai, K., Asayama, K., Campbell, I. A., Sano, S., Kobayashi, S. and Itoh, J., J. Phys. Soc. Japan 40 (1976) 429.

[13] Borg, R. J., Phys. Rev. B 1(1970) 349.

[14] Creuzet, G., Thèse d'Etat, Orsay (1982), unpublished. 